\title{
Dietary change enables robust growth-coupling of heterologous methyltransferase activity in yeast
}

Hansen, A. S. L., ${ }^{1}$ Dunham, M. J., ${ }^{2}$ Arsovska, D., ${ }^{1}$ Zhang, J., ${ }^{1}$ Keasling, J.D., , 3-6 Herrgård, M. J., ${ }^{1,7}$ Jensen, M. K..$^{*}$

${ }^{1}$ Novo Nordisk Foundation Center for Biosustainability, Technical University of Denmark, Kgs. Lyngby, Denmark

2 Department of Genome Sciences, University of Washington, Seattle, Washington 98195 , USA

3 Joint BioEnergy Institute, Emeryville, CA, USA

${ }^{4}$ Biological Systems and Engineering Division, Lawrence Berkeley National Laboratory, Berkeley, CA, USA

${ }^{5}$ Department of Chemical and Biomolecular Engineering \& Department of Bioengineering, University of California, Berkeley, CA, USA

${ }^{6}$ Center for Synthetic Biochemistry, Institute for Synthetic Biology, Shenzhen Institutes of Advanced Technologies, Shenzhen, China

${ }^{7}$ Biolnnovation Institute, Copenhagen, Denmark 
Table S1: Mean vanillic acid (VA) concentrations and optical densities (OD600) for yeast strains expressing OMT variants

\begin{tabular}{|c|c|c|c|}
\hline Strain name & Calc. VA conc. $(\mu \mathrm{M})$ & OD600 & $\begin{array}{c}\text { Normalised conc. } \\
{[\mu \mathrm{M} / \mathrm{OD} 600]}\end{array}$ \\
\hline SCAH138 (empty plasmid) & n.d. & 0,025833 & - \\
\hline SCAH328 (Synechocystis sp. PCC 6803) & 1,658156133 & 0,275333 & 6,137844 \\
\hline SCAH329 (Aquifex aeolicus) & n.d & 0,05 & - \\
\hline SCAH330 (Arabidopsis thaliana) & n.d. & 0,06 & - \\
\hline SCAH331 (Fragaria ananassa) & n.d. & 0,13 & - \\
\hline SCAH332 (Candida orthopsilosis) & 6,354961733 & 0,481667 & 13,18647 \\
\hline SCAH333 (Scheffersomyces stipitis) & 6,820963333 & 0,526333 & 13,37068 \\
\hline SCAH334 (Spathaspora passalidarum) & 11,32155773 & 1,036 & 10,77446 \\
\hline SCAH335 (Chaetomium thermophilum) & n.d. & 0,115333 & - \\
\hline SCAH336 (Yarrowia lipolytica) & 44,89206773 & 3,505667 & 12,83225 \\
\hline SCAH337 (Crenarchaeote) & 21,51227693 & 1,411667 & 15,48475 \\
\hline SCAH338 (Myxosarcina) & 43,94780133 & 3,874 & 11,35764 \\
\hline SCAH339 (Neurospora crassa) & n.d. & 0,113 & - \\
\hline SCAH340 (Thaumarchaeota) & 43,59830013 & 2,233333 & 19,38914 \\
\hline SCAH341 (Phytophthora infestans) & 117,1856756 & 4,658 & 25,15604 \\
\hline SCAH342 (Synechocystis sp. PCC 6714) & 5,2962388 & 0,5525 & 9,583559 \\
\hline SCAH343 (Myxococcus xanthus) & 72,06731893 & 4,285333 & 16,82748 \\
\hline SCAH344 (Human) & 135,8012132 & 4,237167 & 32,07689 \\
\hline
\end{tabular}

Table S2: List of S. cerevisiae strains used in study

\begin{tabular}{|c|c|c|}
\hline Strains & Characteristics & Comment \\
\hline $\begin{array}{l}\text { CEN.PK102- } \\
5 B\end{array}$ & MATa ura3-52 his3 $\Delta 1$ leu2-3/112 & Peter Kötter \\
\hline SCAH100 & CEN.PK102-5B + pRS415U & This study \\
\hline SCAH124 & $\begin{array}{l}\text { MATa ura3-52 his3 } \Delta 1 \text { leu2-3/112 met17 } \Delta \\
\text { cho2 } \Delta \text { opi3 } \Delta \text { met2 } \Delta\end{array}$ & (Luo et al. 2019) \\
\hline SCAH136 & SCAH124+PL_01_D8 & This study \\
\hline
\end{tabular}




\begin{tabular}{|c|c|c|}
\hline SCAH138 & SCAH124+pRS415U & (Luo et al. 2019) \\
\hline SCAH328 & SCAH124+PL_02_F3 & This study \\
\hline SCAH329 & SCAH124+PL_02_F4 & This study \\
\hline SCAH330 & SCAH124+PL_02_F5 & This study \\
\hline SCAH331 & SCAH124+PL_02_F6 & This study \\
\hline SCAH332 & SCAH124+PL_02_F7 & This study \\
\hline SCAH333 & SCAH124+PL_02_F8 & This study \\
\hline SCAH334 & SCAH124+PL_02_F9 & This study \\
\hline SCAH335 & SCAH124+PL_02_G1 & This study \\
\hline SCAH336 & SCAH124+PL_02_G2 & This study \\
\hline SCAH337 & SCAH124+PL_02_G3 & This study \\
\hline SCAH338 & SCAH124+PL_02_G4 & This study \\
\hline SCAH339 & SCAH124+PL_02_G5 & This study \\
\hline SCAH340 & SCAH124+PL_02_G6 & This study \\
\hline SCAH341 & SCAH124+PL_02_G7 & This study \\
\hline SCAH342 & SCAH124+PL_02_G8 & This study \\
\hline SCAH343 & SCAH124+PL_02_G9 & This study \\
\hline SCAH344 & SCAH124+PL_02_E5 & This study \\
\hline SCAH354 & $\begin{array}{l}\text { MATa ura3-52 his3 } \Delta 1 \text { leu2-3/112 met17 } \Delta \\
\text { cho2 } \Delta \text { opi3 } \Delta \text { met2 } \Delta \text { set2 } \Delta \text { dot1 } \Delta \text { set1 } \Delta\end{array}$ & This study \\
\hline SCAH362 & SCAH124+pRS415U & $\begin{array}{l}\text { This study, similar to SCAH138 (Luo et al. } \\
\text { 2019) }\end{array}$ \\
\hline SCAH368 & SCAH354+pRS415U & This study \\
\hline SCAH370 & SCAH124+PL_02_H4 & This study \\
\hline SCAH371 & SCAH354+PL_02_H4 & This study \\
\hline
\end{tabular}

Table S3: List of plasmids used in the study. Sequences of the OMT variants of PL_02_F3PL_02_E5 are listed in Supplementary Table S4. 


\begin{tabular}{|c|c|c|}
\hline Name & Characteristics & Comment \\
\hline PL_01_A9 & CEN/ARS4 ampR HIS3 $\mathrm{P}_{\mathrm{TEF} 1-S p C A S 9-\mathrm{T}_{\mathrm{CYC} 1}}$ & (DiCarlo et al. 2013), this study \\
\hline PL_01_E3 & $2 \mu$ ori, ampR, pESC-URA, gRNA.SET1 & This study \\
\hline PL_01_E6 & $2 \mu$ ori, ampR, pESC-URA, gRNA.SET2-gRNA.DOT1 & This study \\
\hline PL_01_D8 & $\begin{array}{l}\text { CEN/ARS4 ampR pRS415-LEU } \mathrm{P}_{T D H 3} \text { AAAACA-CrLAMT- } \\
\mathrm{T}_{A D H 1}\end{array}$ & This study \\
\hline PL_02_H4 & $\begin{array}{l}\text { CEN/ARS4 ampR pRS415-LEU } \mathrm{P}_{\text {TEF1 AAAACA }} \text {-CaCCS1- } \\
\mathrm{T}_{C Y C 1}\end{array}$ & This study \\
\hline pRS415U & $\begin{array}{l}\text { CEN/ARS4 ampR pRS415-LEU2 } \mathrm{T}_{\mathrm{ADH} 1}<- \text {-USER cassette- } \\
>\mathrm{T}_{\mathrm{CYC} 1}\end{array}$ & (E. D. Jensen et al. 2017) \\
\hline PL_02_E5 & $\begin{array}{l}\text { CEN/ARS4 ampR pRS415-LEU P } \mathrm{P}_{T E F 1} \text { AAAACA }-H S O M T \text { - } \\
\mathrm{T}_{C Y C 1}\end{array}$ & $\begin{array}{l}\text { This study, HsOMT cloned from } \\
\text { pXII2-54 in (Strucko, } \\
\text { Magdenoska, and Mortensen } \\
\text { 2015) }\end{array}$ \\
\hline PL_02_F3 & $\begin{array}{l}\text { CEN/ARS4 ampR pRS415-LEU } \mathrm{P}_{\text {TEF1 AAAACA }} \text {-SyOMT- } \\
\mathrm{T}_{C Y C 1}\end{array}$ & This study \\
\hline PL_02_F4 & $\begin{array}{l}\text { CEN/ARS4 ampR pRS415-LEU } \mathrm{P}_{\text {TEF1 AAAACA }} \text {-AaOMT- } \\
\mathrm{T}_{C Y C 1}\end{array}$ & This study \\
\hline PL_02_F5 & $\begin{array}{l}\text { CEN/ARS4 ampR pRS415-LEU P } \mathrm{P}_{\text {TEF1 AAAACA }} \text {-AtOMT- } \\
\mathrm{T}_{C Y C 1}\end{array}$ & This study \\
\hline PL_02_F6 & $\begin{array}{l}\text { CEN/ARS4 ampR pRS415-LEU } \mathrm{P}_{\text {TEF1 AAAACA }} \text {-FaOMT- } \\
\mathrm{T}_{C Y C 1}\end{array}$ & This study \\
\hline PL_02_F7 & $\begin{array}{l}\text { CEN/ARS4 ampR pRS415-LEU } \mathrm{P}_{\text {TEF1 AAAACA }} \text {-COOMT- } \\
\mathrm{T}_{C Y C 1}\end{array}$ & This study \\
\hline PL_02_F8 & $\begin{array}{l}\text { CEN/ARS4 ampR pRS415-LEU } \mathrm{P}_{T E F 1} \text { AAAACA-SsOMT- } \\
\mathrm{T}_{C Y C 1}\end{array}$ & This study \\
\hline PL_02_F9 & $\begin{array}{l}\text { CEN/ARS4 ampR pRS415-LEU } \mathrm{P}_{\text {TEF1 AAAACA }} \text {-SpOMT- } \\
\mathrm{T}_{C Y C 1}\end{array}$ & This study \\
\hline PL_02_G1 & $\begin{array}{l}\text { CEN/ARS4 ampR pRS415-LEU P } \mathrm{P}_{\text {TEF1 AAAACA }} \text {-CtOMT- } \\
\mathrm{T}_{C Y C 1}\end{array}$ & This study \\
\hline PL_02_G2 & 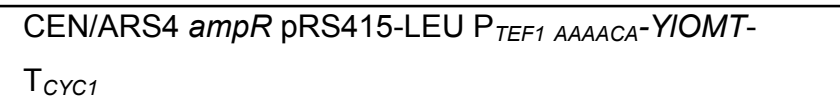 & This study \\
\hline PL_02_G3 & $\begin{array}{l}\text { CEN/ARS4 ampR pRS415-LEU } \mathrm{P}_{\text {TEF1 AAAACA }} \text {-CrOMT- } \\
\mathrm{T}_{C Y C 1}\end{array}$ & This study \\
\hline PL_02_G4 & $\begin{array}{l}\text { CEN/ARS4 ampR pRS415-LEU } \mathrm{P}_{\text {TEF1 AAAACA }}-M Y O M T- \\
\mathrm{T}_{C Y C 1}\end{array}$ & This study \\
\hline PL_02_G5 & $\begin{array}{l}\text { CEN/ARS4 ampR pRS415-LEU } \mathrm{P}_{\text {TEF1 AAAACA }}-\mathrm{NCOMT-} \\
\mathrm{T}_{C Y C 1}\end{array}$ & This study \\
\hline
\end{tabular}




\begin{tabular}{|c|c|c|}
\hline PL_02_G6 & $\begin{array}{l}\text { CEN/ARS4 ampR pRS415-LEU } \mathrm{P}_{\text {TEF1 AAAACA }} \text {-ThOMT- } \\
\mathrm{T}_{\text {CYC1 }}\end{array}$ & This study \\
\hline PL_02_G7 & $\begin{array}{l}\text { CEN/ARS4 ampR pRS415-LEU P } P_{\text {TEF1 AAAACA }} \text { PIOMT- } \\
T_{C Y C 1}\end{array}$ & This study \\
\hline PL_02_G8 & $\begin{array}{l}\text { CEN/ARS4 ampR pRS415-LEU P PEF1 AAAACA- } \\
\text { Sy6714OMT-T }{ }_{C Y C 1}\end{array}$ & This study \\
\hline PL_02_G9 & $\begin{array}{l}\text { CEN/ARS4 ampR pRS415-LEU P PEF1 AAAACA-MyXOMT- } \\
T_{C Y C 1}\end{array}$ & This study \\
\hline
\end{tabular}

Table S4: List of MTase sequences (originally ordered as synthetic gBlock sequences). The sequences have been codon optimized to $S$. cerevisiae using the IDT web service except HsOMT and CrLAMT. Start and stop codon is underlined. All MTases were selected from Kunjapur \& Prather, ACS Synthetic Biology, Sep 20;8(9):1958-1967 (2019).

\begin{tabular}{|c|c|c|c|c|}
\hline Plasmid & MTase ID & Nucleotide sequence & $\begin{array}{l}\text { Original } \\
\text { species }\end{array}$ & $\begin{array}{l}\text { NCBI } \\
\text { Accession }\end{array}$ \\
\hline PL_02_H4 & CaCCS1 & $\begin{array}{l}\text { ATGGAGTTGCAAGAGGTTTTGCACATGAATGGT } \\
\text { GGGGAAGGGGACACATCTTATGCTAAAAACAGT } \\
\text { TCCTACAATCTATTTTTGATTCGTGTCAAACCTG } \\
\text { TCTTAGAACAGTGCATTCAGGAACTATTGAGAG } \\
\text { CGAACCTGCCGAACATAAATAAATGCTTTAAGGT } \\
\text { AGGAGACTTAGGTTGCGCGAGTGGTCCAAACAC } \\
\text { CTTTTCCACAGTAAGGGATATTGTTCAAAGTATA } \\
\text { GACAAAGTAGGACAAGAGAAAAAAACGAACTA } \\
\text { GAGAGACCGACAATTCAAATTTTCCTGAACGATT } \\
\text { TGTTCCAAAATGACTTCAATTCCGTTTCAAGCT } \\
\text { TTTGCCGTCCTTTTACCGTAACTTGGAAAAAGA } \\
\text { AACGGTCGTAAAATCGGTTCTTGTCTGATAGGA } \\
\text { GCAATGCCTGGGTCTTTCTACTCTCGTCTGTTC } \\
\text { CCTGAGGAGAGTATGCACTTTTTACACTCATGCT } \\
\text { ATTGTTTACACTGGTTATCACAAGTACCATCAGG } \\
\text { CTTAGTAACTGAGCTTGGTATTTCTGCCAATAAG } \\
\text { GGGTGTATCTATTCCTCCAAGGCCTCCGGTCCG } \\
\text { CCCATCAAGAAAGCGTACCTTGACCAATTCACT } \\
\text { AAAGATTTCACCACGTTCTTGCGTATTCACAGCG } \\
\text { AAGAGCTTATCAGCCGTGGCAGGATGCTTCTAA } \\
\text { CATTTATTTGTAAAGAAGACGAATTCGATCATCC } \\
\text { CAACTCCATGGATCTTCTAGAGATGTCTATCAAC } \\
\text { GATTTGGTAATTGAGGGTCATCTGGAAGAAGAG } \\
\text { AAGCTTGATAGTTTTAACGTTCCGATTTACGCAC } \\
\text { CGAGCACAGAAGAGGTGAAACGATTGTCGAG } \\
\text { GAAGAGGGTAGTTTTGAGATTCTATATCTTGAAA } \\
\text { CTTTCTACGCCCCGTATGACGCCGGTTTCAGCA }\end{array}$ & $\begin{array}{l}\text { Coffea } \\
\text { arabica }\end{array}$ & \\
\hline
\end{tabular}




\begin{tabular}{|c|c|c|c|}
\hline & & $\begin{array}{l}\text { TTGATGACGACTATCAAGGACGTTCACATTCAC } \\
\text { CAGTCTCCTGCGATGAGCATGCCCGTGCCGCC } \\
\text { CACGTGGCCAGCGTGGTGCGTTCAATATATGAG } \\
\text { CCCATACTGGCTTCCCACTTCGGTGAAGCAATT } \\
\text { CTGCCCGATTTGTCTCACCGTATTGCAAAGAAT } \\
\text { GCCGCCAAGGTGCTAAGGTCCGGGAAAGGGTT } \\
\text { CTATGACAGCGTGATAATTAGCCTTGCCAAAAAA } \\
\text { CCTGAGAAGGCCGATATGTAA }\end{array}$ & \\
\hline PL_01_D8 & CrLAMT & $\begin{array}{l}\text { ATGGTAGCAACGATTGATTCAATTGAAATGCCTG } \\
\text { CCTTACCAACTGCGGTCGAAGCTCACCCCATGA } \\
\text { AAGGTGGTGATGATTCTCATTCCTATAGCCAGA } \\
\text { ATAGTTGTTACCAGAAAGGTGTCATTGATGCGG } \\
\text { CCAAGGCTGTCATTGTTGAGGCCGTCAATGAAA } \\
\text { AACTGGATCTAGAAAATAATCCAATTTTGATCC } \\
\text { TATTAAACCTTTCAGGATTGCTGATTTCGGTTGC } \\
\text { TCCACGGGTCCTAATACTTTTCATGCCATGCAAA } \\
\text { ATATTGTTGAATCCGTAGAAACAAAGTATAAATC } \\
\text { CCTTCAAAAGACCCCTGAGTTTCATGTCTTTTTT } \\
\text { AATGATCACGTCAACAATGATTTTAACGTCTTAT } \\
\text { TTCGTTCTCTTCCGCCCAATAGGGAGTTTTTCGC } \\
\text { GGCTGGTGTACCAGGTTCATTTTATACAAGGGT } \\
\text { ATTCCCAAAGAATAGTATTCATTTTGCCCACTGT } \\
\text { TCTTACGCATTGCATTGGCTATCTAAGGTTCCTA } \\
\text { AGGAAATTCAAGACAAAAACTCTTTGGCGTACAA } \\
\text { TAAGGGTAGGATTCATTACACTGGCACTGAGAA } \\
\text { ACACGTAGTCAAAGCTTACTTTGGTCAATTCCAG } \\
\text { AGAGATTTCGAAGGCTTTCTAAAAGCTAGAGCC } \\
\text { CAGGAGATAGTCGTGGGTGGTTTAATGGTTATA } \\
\text { CAAATACCGGGCTTACCTTCCGGAGAAGTGCTA } \\
\text { TTCAGTAGAACTGGTGCAGGTCTATTGCATTTCT } \\
\text { TACTAGGTACGAGCTTAATGGAGTTGGTAAATAA } \\
\text { GGGTATAATCAACGAAGAAAGCGTTGACAGCTT } \\
\text { CAACTTACCGCAATATCATCCCTCAGTTGAAGAC } \\
\text { TTGGAAATGGTTATAGAAATGAACGATTGCTTCA } \\
\text { CAATTGAGAGAGTCGGCACATTGCCCCATCCAA } \\
\text { TGAAGAACCTACCTTTCGATGTCCAAAGAACATC } \\
\text { TTTACAAGTGAGAGCAATCATGGAATGTATTCTT } \\
\text { ACTGAACATTTTGGCGAGAACATCTTGGATCCA } \\
\text { CTATTCGAAATCTACACTAAGAACCTACAAGAGA } \\
\text { ACTTCCATGTTTTCGATAAGGAAATCAGAAAGGA } \\
\text { TGCAGATTTGTACCTTGTTTTGAAGAGAAAAGG } \\
\text { GAACTAA }\end{array}$ & $\begin{array}{l}\text { Catharan } \\
\text { thus } \\
\text { roseus }\end{array}$ \\
\hline
\end{tabular}




\begin{tabular}{|c|c|c|c|c|}
\hline PL_02_E5 & HsOMT & $\begin{array}{l}\text { ATGGGTGACACTAAGGAGCAAAGAATTTTGAAC } \\
\text { CATGTTTTGCAACATGCTGAGCCAGGTAACGCT } \\
\text { CAATCTGTTTTGGAGGCTATTGACACTTATTGTG } \\
\text { AGCAAAAGGAGTGGGCTATGAACGTTGGTGACA } \\
\text { AGAAGGGTAAAATTGTTGACGCTGTTATTCAAGA } \\
\text { GCATCAACCATCTGTTTTGTTGGAGTTGGGTGC } \\
\text { TTATTGTGGTTATTCAGCTGTTAGAATGGCTAGA } \\
\text { TTGTTGTCACCAGGTGCTAGATTGATTACTATTG } \\
\text { AGATTAACCCAGACTGTGCTGCTATTACTCAAAG } \\
\text { AATGGTTGATTTCGCTGGTGTTAAGGACAAGGT } \\
\text { TACTTTGGTTGTTGGTGCTTCTCAAGACATTATT } \\
\text { CCACAATTGAAGAAGAAGTATGATGTTGACACTT } \\
\text { TGGACATGGTTTTCTTGGACCATTGGAAGGACA } \\
\text { GATATTTGCCAGACACTTTGTTGTTGGAGGAGT } \\
\text { GTGGTTTGTTGAGAAAGGGTACTGTTTTGTTAG } \\
\text { CTGACAACGTTATTTGTCCAGGTGCTCCAGACT } \\
\text { TCTTGGCTCATGTTAGAGGTTCTTCATGTTTCGA } \\
\text { GTGTACTCATTATCAATCTTTCTTGGAATATAGA } \\
\text { GAGGTTGTTGACGGTTTGGAGAAGGCTATATAT } \\
\text { AAGGGTCCAGGTTCTGAAGCTGGTCCATAA }\end{array}$ & Human & - \\
\hline PL_02_F3 & SyOMT & $\begin{array}{l}\text { ATGGGTAAAGGCATAACTGGATTTGACCCATC } \\
\text { TCTTTACTCTTACCTACAGAGCATCAGCGCGG } \\
\text { ACGACAGCTTTTATTTGGCTCAGTTAAGACGTG } \\
\text { AAACGGCCCATCTACCCGGTGCCCCTATGCAG } \\
\text { ATCAGCCCTGAGCAGGCACAATTTCTTGGCTT } \\
\text { GCTAATAAGTCTAACAGGAGCGAAACAGGTCT } \\
\text { TGGAAATAGGGGTATTCAGAGGTTACTCTGCC } \\
\text { TTGGCTATGGCGCTTCAGCTACCGCCCGACG } \\
\text { GACAAATTATAGCTTGCGACCAAGACCCCAAT } \\
\text { GCTACAGCCATAGCGAAGAAATACTGGCAAAA } \\
\text { AGCAGGAGTCGCAGAGAAGATCAGCCTAAGA } \\
\text { CTGGGACCCGCTCTAGCAACACTGGAACAACT } \\
\text { GACCCAAGGTAAGCCACTACCGGAGTTTGATC } \\
\text { TTATCTTTATCGACGCTGACAAGAGAAATTATC } \\
\text { CACGTTATTATGAAATAGGACTAAACCTTCTTA } \\
\text { GAAGAGGGGGTTTAATGGTAATTGACAATGTG } \\
\text { TTGTGGCATGGAAAAGTGACAGAAGTTGACCC } \\
\text { CCAGGAAGCCCAGACGCAAGTTCTACAACAGT } \\
\text { TTAACCGTGATCTGGCACAGGATGAAAGAGTA } \\
\text { CGTATTAGTGTTATACCCTTGGGCGATGGAAT } \\
\text { GACGCTAGCACTTAAAAAGTAA }\end{array}$ & $\begin{array}{l}\text { Synech } \\
\text { ocystis } \\
\text { sp. } \\
\text { PCC } \\
6803\end{array}$ & $\begin{array}{l}\text { BAA1056 } \\
7.1\end{array}$ \\
\hline PL_02_F4 & AaOMT & $\begin{array}{l}\text { ATGGCGTTTATCTGCCCGGAGGAGATAGAAGC } \\
\text { ATACGCCGAGGAGCATAGCTCAGAGTTGCATC } \\
\text { CGATATTGGAGGAACTAGTAGAGTTCACCTAT }\end{array}$ & $\begin{array}{l}\text { Aquifex } \\
\text { aeolicu } \\
s\end{array}$ & $\begin{array}{l}\text { AAC0743 } \\
5.1\end{array}$ \\
\hline
\end{tabular}




\begin{tabular}{|c|c|c|c|c|}
\hline & & $\begin{array}{l}\text { AAGAATACTGACCTTCCACAGATGATGGTAGG } \\
\text { CAGGGCTGAAGGAAACTTTTTGAAGATGTTGG } \\
\text { TCGAGATATCAGGAGCAAAAAGGGTTTAGAA } \\
\text { ATAGGTACGTTTACAGGGTTTAGTGCCTTGAT } \\
\text { GATGGCACAAGGGCTACCAGAGGATGGAAAG } \\
\text { TTGACAACTATTGAGGTCAATCCGGAATATGCT } \\
\text { CAAATGGCGAAATCTTTCATAGAACGTGCTCC } \\
\text { GTGGGGCAAAAAAATTGAAGTGATTGAGGGG } \\
\text { ACGCTAGGAAGGTGTTAGAGGAATTAAGAAA } \\
\text { GAGAGCTTTGACTTTATCTTTATTGATGCCGAC } \\
\text { AAGTCTTCATACCCCTACTATTACGAAAAATGT } \\
\text { TTAGAGTTACTAAAAAAAGGCCGTTTGATGGCA } \\
\text { ATAGATAACGCTCTATGGGAGGGCAAAGTATT } \\
\text { GGAACCTGATGACAATAGGTCTAAAGCAATCG } \\
\text { CTAGGATGAACGAAATGATAAAAGAGGATCCC } \\
\text { AGAGTAGAGAAAGTGCTATTGACAGTTAGGGA } \\
\text { TGGGATTTTCCTGGTTCGTAAGATCTAA }\end{array}$ & & \\
\hline PL_02_F5 & AtOMT & $\begin{array}{l}\text { ATGGGATCAACAGCCGAGACGCAGCTTACTCC } \\
\text { GGTACAAGTCACCGACGACGAGGCGGGCTT } \\
\text { TTTGCGATGCAGTTGGCATCTGCCAGTGTTTTA } \\
\text { CCCATGGCACTAAAAAGTGCATTGGAATTGGA } \\
\text { CCTGCTGGAAATCATGGCGAAAAACGTAGCC } \\
\text { CCATGAGTCCTACCGAGATAGCCAGCAAATTG } \\
\text { CCGACCAAGAACCCAGAAGCACCGGTTATGCT } \\
\text { TGACAGGATTCTGCGTTTGTTGACATCATATTC } \\
\text { TGTCCTGACATGCTCTAACAGAAAGCTATCAG } \\
\text { GGGATGGGGTTGAAAGGATTTACGGGCTTGG } \\
\text { GCCAGTTTGTAAATATCTTACCAAAAATGAGGA } \\
\text { CGGTGTGTCAATCGCTGCACTGTGTCTTATGA } \\
\text { ATCAAGACAAAGTTCTTATGGAGAGCTGGTAT } \\
\text { CATTTGAAAGACGCTATACTTGATGGAGGCATT } \\
\text { CCGTTCAATAAGGCGTATGGAATGAGCGCATT } \\
\text { TGAGTACCACGGCACGGATCCAAGATTCAATA } \\
\text { AAGTCTTCAACAACGGTATGAGCAATCATTCTA } \\
\text { CCATTACCATGAAGAAAATCCTAGAAACTTACA } \\
\text { AAGGTTTTGAGGGTCTAACCTCCCTGGTCGAC } \\
\text { GTAGGCGGAGGAATTGGGGCTACACTGAAGA } \\
\text { TGATTGTCAGTAAATATCCAAACTTAAAGGGAA } \\
\text { TAAATTTTGACCTACCTCATGTAATTGAGGATG } \\
\text { CGCCCTCACACCCGGGAATTGAGCATGTTGGT } \\
\text { GGTGATATGTTCGTGAGTGTTCCCAAGGGCGA } \\
\text { CGCCATATTTATGAAGTGGATATGTCACGACT } \\
\text { GGTCCGATGAGCATTGCGTAAAGTTCCTAAAA } \\
\text { AATTGTTATGAAAGTCTTCCCGAAGACGGCAA }\end{array}$ & $\begin{array}{l}\text { Arabido } \\
\text { psis } \\
\text { thalian } \\
a\end{array}$ & $\begin{array}{l}\text { AAL3291 } \\
5.1\end{array}$ \\
\hline
\end{tabular}




\begin{tabular}{|c|c|c|c|c|}
\hline & & $\begin{array}{l}\text { AGTGATTCTGGCGGAATGCATCCTTCCTGAAA } \\
\text { CACCCGATTCCAGCTTATCCACTAAACAGGTA } \\
\text { GTGCATGTCGATTGTATAATGCTGGCGCACAA } \\
\text { TCCGGGCGGCAAAGAGAGGACTGAGAAGGAA } \\
\text { TTTGAAGCGCTAGCTAAGGCGTCAGGTTTTAA } \\
\text { AGGGATAAAGGTGGTCTGCGATGCATTTGGCG } \\
\text { TCAATTTGATAGAACTGCTGAAGAAATTGTAA }\end{array}$ & & \\
\hline PL_02_F6 & FaOMT & $\begin{array}{l}\text { ATGGGTTCTACCGGGGAGACGCAAATGACTCC } \\
\text { GACCCATGTTTCTGACGAGGAAGCCAATTATT } \\
\text { CGCAATGCAGTTGGCAAGCGCTTCAGTATTGC } \\
\text { CAATGGTACTTAAGGCTGCTATTGAACTGGAT } \\
\text { CTTCTTGAGATAATGGCTAAGGCTGGCCCCGG } \\
\text { ATCATTTCTTTCCCCGAGCGATCTGGCGAGCC } \\
\text { AGCTTCCTACGAAAATCCAGAAGCTCCTGTG } \\
\text { ATGCTAGACCGTATGCTTAGGCTTCTAGCTTCT } \\
\text { TACTCCATCTTAACGTGTTCATTAAGAACCTTG } \\
\text { CCCGATGGTAAAGTCGAACGTCTTTACTGTTT } \\
\text { GGGCCCGGTGTGTAAGTTCTTGACAAAAAATG } \\
\text { AGGACGGTGTGTCTATTGCAGCGTTATGCTTA } \\
\text { ATGAATCAGGACAAGGTGTTAGTTGAGAGCTG } \\
\text { GTACCATCTGAAGGATGCGGTCCTAGATGGCG } \\
\text { GAATCCCGTTTAACAAAGCATATGGCATGACA } \\
\text { GCCTTCGATTATCACGGAACGGACCCGAGATT } \\
\text { TAATAAAGTTTTCAACAAAGGGATGGCAGACC } \\
\text { ACTCCACTATCACTATGAAGAAGATCCTGGAAA } \\
\text { CTTACAAAGGCTTCGAGGGACTAAAGAGCATT } \\
\text { GTAGATGTTGGAGGAGGTACAGGTGCAGTGG } \\
\text { TCAACATGATTGTTTCCAAGTATCCGTCAATCA } \\
\text { AAGGTATTAATTTCGATTTGCCTCATGTTATCG } \\
\text { AGGATGCGCCGCAGTACCCGGGGGTTCAGCA } \\
\text { CGTCGGGGGTGATATGTTCGTTAGTGTTCCCA } \\
\text { AGGGAAATGCGATCTTCATGAAATGGATCTGC } \\
\text { CACGATTGGTCCGATGAACATTGCATAAAATTT } \\
\text { TTAAAGAACTGTTATGCGGCCCTACCTGACGA } \\
\text { CGGAAAAGTTATATTGGCCGAGTGTATCCTTC } \\
\text { ATAA } \\
\text { GGATGGCACCTGATACCTCTTTAGCTACCAAG } \\
\text { ACATAACCCAGGCGGAAAGGAAAGGACTGAAC }\end{array}$ & $\begin{array}{l}\text { Fragari } \\
\text { a } \\
\text { ananas } \\
\text { sa }\end{array}$ & $\begin{array}{l}\text { AAF2835 } \\
3.1\end{array}$ \\
\hline$\overline{P L}$ _02_F7 & COOMT & $\begin{array}{l}\text { ATGTCCACTAGCAATACGGCGGAAAAGACCAA } \\
\text { GGAGCAGAAGTTCCATGAGTACATTTTAAATCT }\end{array}$ & $\begin{array}{l}\text { Candid } \\
\text { a }\end{array}$ & $\begin{array}{l}\text { CCG2504 } \\
7.1\end{array}$ \\
\hline
\end{tabular}




\begin{tabular}{|c|c|c|c|c|}
\hline & & $\begin{array}{l}\text { TCCCCAGGATAAGGTAGACGCCATCAAGGGTA } \\
\text { AGCCATTAGAGGTGCTAAAGGTTATTGACGAC } \\
\text { TACGGCGAGCATTTTATGGACATCGGCAGGCA } \\
\text { AAAAGGTGAGCTAATAACGACAGAAATCAGAG } \\
\text { AGAAGAAACCCAAAGTAATGATTGAGTTGGGT } \\
\text { GGCTATCTGGGTTACTCAGCAGTTCTGTTTGC } \\
\text { AAACGAGCTTATTCAAGATCCGGAGGCCAAAT } \\
\text { ATTATTCTTTCGAGGTAAACCCGGAATTTGCCA } \\
\text { AGATTGCCACGGACGTCATCAGGCTGGCGGG } \\
\text { GCTGTCAAAAAAAATCGAAATAATCGTCGGTAA } \\
\text { AGCGTCCTACACGTTAGTCGACTTCAAAGAGA } \\
\text { GGCTTAACAAAAACGGTTCTTTTAAAGCACTTG } \\
\text { ATTTTATATTCATAGATCACTGGAAAGATTTGTA } \\
\text { CGTCCCAGATTTGAGAGAGCTAGAGTCTCTTA } \\
\text { ATTTTATCGCACCGGGGACTGTCATCACTGCC } \\
\text { GACAACATTTTAACTCCGGGTGCGCCCGAGTA } \\
\text { TGCAAAATACGTCCGTTTGTCTCCAGAGGAGA } \\
\text { AGAAGGAATATAATGAAAAAAACCCAAACCCTA } \\
\text { ATGGGGACGAGTTCCTGGGGAGATGGAACAT } \\
\text { ACTGTACGACACCGACACCATTAAAGTCGAGT } \\
\text { ATCCGAACGGTCATGTGGATGCCGTTGAAATA } \\
\text { ACGAAATGTACTGACTACCTTTCTGGATAA }\end{array}$ & $\begin{array}{l}\text { orthops } \\
\text { ilosis }\end{array}$ & \\
\hline PL_02_F8 & SsOMT & $\begin{array}{l}\text { ATGCCAGAGAAGGAGCAAAAGGTCTTGCAACA } \\
\text { TATCTTGAGTTTACCTGAGGATAGGCTGAAGC } \\
\text { AAATCAGAGGAAAGCACAGGAAGTACTAAAG } \\
\text { CTTATTGATGATTTCAGTGAGAATGTTGAGAGC } \\
\text { TTGATGAATATCGGACCTTATAAAGGTAAGTTA } \\
\text { ATAGTCGATAAGATTAAGGAAAAGAACCTAAG } \\
\text { ATAATGGTGGAGCTGGGGGGTTATGTCGGGTA } \\
\text { TTCCGCGATATTGTTTGGCTCAAGTCTACCCG } \\
\text { ACGATCCGTCCTCTCATTACTACAGCTTAGAAC } \\
\text { TAAATGAAGAGTTCGCCAACATCTCTAGGCAA } \\
\text { CTAATTGACCTAGCTGGTCTTTCCCATAGGGT } \\
\text { GTCAATCATAGTGGGTAAGGCCAGTTCCAGTC } \\
\text { TAGTAGAGATGGTAGATCATTTTGAGGAGGGA } \\
\text { AACAAAGGGTACTTCAGTTTCGACTTTATTTTC } \\
\text { ATTGACCACTGGAAAGACATGTACGTTCCTGA } \\
\text { TCTTAGAGTATTGGAGTCCCTAACACTAATCTC } \\
\text { CCCTGGAACAATCATAGTCGCTGACAATATTTA } \\
\text { TTACCCCGGAGCGCCAGATTATGTAAAGTACA } \\
\text { TTCAGGGCAGTCCAGAGGAGAGGAGAGACCA } \\
\text { CAATTACACTGTCACTAATGTAGCTGCCCCTGA } \\
\text { ATTCCAAGGACGTTGGAACATTTTGTATAAGAG } \\
\text { CGAGACAATACCCGTGAAAAACCCTAAATCTG }\end{array}$ & $\begin{array}{l}\text { Scheffe } \\
\text { rsomyc } \\
\text { es } \\
\text { stipitis }\end{array}$ & $\begin{array}{l}\text { ABN6792 } \\
1.2\end{array}$ \\
\hline
\end{tabular}




\begin{tabular}{|c|c|c|c|c|}
\hline & & $\begin{array}{l}\text { GAACCGAGGATGCTATAGAAGTTACCGAGTGT } \\
\text { GTAGAGTACTTAAATGGTIAA }\end{array}$ & & \\
\hline PL_02_F9 & SpOMT & $\begin{array}{l}\text { ATGACAGACCAAAGTAAAGAGGAGAAACTGGC } \\
\text { CGAATACGTGTTGACCCACCCGCAAGTAGAAA } \\
\text { GTGCGCGTGGGAAGCCTAGAGAAGTCCTAAG } \\
\text { ACTTATTGAAGAGTACGATTCCAAAACTCAGCT } \\
\text { TGAAATGATCATAGGTCCCCATAAGGGACAAG } \\
\text { TCATCATTGATGAGATTAAAAAGGTGAATCCGA } \\
\text { AAATTATGATCGAGCTAGGGTGCTATGTAGGA } \\
\text { TATTCTGCGATTCTGTTTGCTTCCGTTTACCA } \\
\text { GAAGATTCAAAGTATTATAGCTTTGAACTAAAC } \\
\text { GACAAGTTCGCAGCCATAGCTAGGAAATTTAT } \\
\text { ATCTTTAGCTGGCCTTGATCATAAGATAGAAAT } \\
\text { ATTTGTTGGAAAGGCCTCCGACAGGCTGCCAG } \\
\text { AGTTCAGGGATAAAATAGGAAACGTCAACGGG } \\
\text { AAGCCGAAAGCGGTAGAGTTTGTATTTATCGA } \\
\text { TCATTGGAAGGACGTTTATGTGCCCGACCTGA } \\
\text { GAATTTTGGAGACGCTAAATTTCATAGCTCCG } \\
\text { GGCACCGTTATTGCTGCCGACAACATTATCTAT } \\
\text { CCTGGGGTCCCAGGATACACCGATTACGTGCA } \\
\text { GGGTAGTGCCGAATATAAGAAAGAACACAACA } \\
\text { AGACTAACACCAACAAAAACGGAGCGGAGTAT } \\
\text { GTGGGGCGTTGGAATATCGTTTATAAATCAGA } \\
\text { AACTATCGAAGTCGAGAACCCTAATCGTCATA } \\
\text { GAGACGGGGTGGAGATAAGCAGGTGCGTGGA } \\
\text { TGTTCTAGCAGAGTAA }\end{array}$ & $\begin{array}{l}\text { Spatha } \\
\text { spora } \\
\text { passali } \\
\text { darum }\end{array}$ & $\begin{array}{l}\text { EGW299 } \\
58.1\end{array}$ \\
\hline $\begin{array}{l}\mathrm{PL}+02 \_\mathrm{G} \\
1\end{array}$ & CtOMT & $\begin{array}{l}\text { ATGTCCACGCCCGCGTTTGATCCCACAAAGGC } \\
\text { ATACAAAAGATTTGAAGTAAACGGCGCTTTTCT } \\
\text { AGGAGACGGAAGGGAGGTGGCCCTGTTAGAA } \\
\text { TATGTCTATAGTAGGCCAGATTTGGACCAACT } \\
\text { GAGGGGAAACCCTTCCAGAGTGTTACAAGCCA } \\
\text { TAGATGAATTTGCTAGAACACACACAGGTTTAA } \\
\text { TAAATGTCGGTGAGATAAAGGGCGCGATAGTC } \\
\text { TGCGACACGATAGCCAAATACAAACCGGCCAT } \\
\text { CATGGTGGAATTAGGCTCATACGTAGGATACT } \\
\text { CAGCAATTCTGTTTGGAAACGCGTTAAAGAAA } \\
\text { GCTGGTGGAAAAGCTTACTACTCAATCGAGAA } \\
\text { AAGTCCCCTGTTTGCGGCCATCTCTACCTCATT } \\
\text { AGTAGACTTAGCCGGATTGCGTGATACCGTCC } \\
\text { GTGTCATTGTGGGGACGGGAGAGGAAGGAAT } \\
\text { AATGAGACTTCACAAAGAAGGAGTTCTGGAAC } \\
\text { CAAAAGTGGATATGTTTTTCTTTGATCATCATA } \\
\text { AACCCAGCTATACCCCTGATCTGAAAGTATGT } \\
\text { GAGCGTCTGGGCCTAGTGGGAGTTGGAACAG }\end{array}$ & $\begin{array}{l}\text { Chaeto } \\
\text { mium } \\
\text { thermo } \\
\text { philum }\end{array}$ & $\begin{array}{l}\text { EGS2186 } \\
3.1\end{array}$ \\
\hline
\end{tabular}




\begin{tabular}{|c|c|c|c|c|}
\hline & & $\begin{array}{l}\text { TGTTAGTAGCTGATAATATGATCCAGCCTGGG } \\
\text { AACCCAGCCTATATGGCCTATGTAAGGGCTTC } \\
\text { CGTAGCCGAAAAAAGGGAGCTAGACAAGGAAA } \\
\text { GCACTGAAAAAGGAAACCCTAATCTTCAATACA } \\
\text { GAAGTCAGTTCATAAAAAGCTTTGATCCTTCTG } \\
\text { GTGATGAGGATGCGTTGGAAATTACTGAATGC } \\
\text { GTCGGGGTGGAAGCGTAA }\end{array}$ & & \\
\hline $\begin{array}{l}\overline{P L}{ }_{-} 02 \_G \\
2\end{array}$ & YIOMT & $\begin{array}{l}\text { ATGAGTACAACATATAAGGAATACGACTGGGA } \\
\text { AAAACCCGTTCAGTTGTTGTATGACCATATAAA } \\
\text { CGGGTTACCAGCAGAAGAACTGGCTAAATTCA } \\
\text { AGAAAGATCCAGAAGCCTGCTAGACGCCATT } \\
\text { GACAAGTTTGAGGAGGAGCAACACAAGAAAGA } \\
\text { CGGGAAATACGCTCTAATGACAGTGGGCCCG } \\
\text { GAGAAAAGGAAGGTAGTAGAGGAAGAATTGC } \\
\text { ACAGAACAAACCTTACACGTTTGCAGAGCTAG } \\
\text { GTGGCTTCTGTGGATATTCAGCTATTGCGTTC } \\
\text { GCTCATAAGCTAAAGCAGACACATCCTGGTTA } \\
\text { CCAAGTCCATTATTACAGCCTGGAGGTTTCCC } \\
\text { CGTTTTTCGCCAAGGTCACATCCAGGTTTACG } \\
\text { ACACTGGCGGGATTAGGCGACATTGTTACAGT } \\
\text { ATTAGTAGGCCCAGCTGCGGAAGGATTAGAGC } \\
\text { GTTTGAAAGAGGAGTACAATCATGCGAAGATT } \\
\text { GACTTCTTCTTCATAGACCACTGGAAAGACTTG } \\
\text { TATGTCCCAGATTTGCAGAAAGCAGAAGAGTT } \\
\text { GGGACTAGTACAGTACGGAACGGTCATAACTG } \\
\text { CGGATAATATCTACTGGCCCGGGGCGCCTGA } \\
\text { GTATGCGAAGTACGTAAGGCTGACACCGGCC } \\
\text { GAAAAAAAGAAAGAATATCCGGGTAAAGGAAA } \\
\text { CCCAAACATAGAGTACGATACTAGAACCGTCG } \\
\text { AAGTACCTTTAGGCAAGGACAAAAAGGACGGG } \\
\text { GTAGAGGTCACACTGTGCACAGGCGTGCTTAC } \\
\text { CGAATAA }\end{array}$ & $\begin{array}{l}\text { Yarrowi } \\
a \\
\text { lipolytic } \\
\text { a }\end{array}$ & $\begin{array}{l}P_{5} 5004 \\
51.1\end{array}$ \\
\hline $\begin{array}{l}\overline{P L}+02 \_G \\
3\end{array}$ & CrOMT & $\begin{array}{l}\text { ATGATAAATGAAAAAATACAGTACGTGTTGTCT } \\
\text { AGGCTGGAAAAAGATATAGACTACGAAACTAA } \\
\text { ACATTTGGACGAAATTCCCTCCGAGAAGCGTC } \\
\text { TAAACTGCATATCTAAGAACATCGGCATGTTTT } \\
\text { ACAACATATTACTGAAGAGTATAAACGCCAAGA } \\
\text { ATATTTTAGAAATAGGGATGTCTGTTGGTTATT } \\
\text { CAGGGCTGTGGTTCGCGGATGCTGTCATGAGT } \\
\text { AATAAGCAGCTAAACGGACAGATCATTACGATT } \\
\text { GACAGGGAGAAATTCAAAATCGATTCCGCTAC } \\
\text { GAGAAATTTTGAAGAAGCAGGCGTAGGCTCAC } \\
\text { TAATCAAAATCCGTGAGGGGGAGGCAAGAAAA } \\
\text { ATATTACATGAAATCAAAGAAGAGTTTGGGGA }\end{array}$ & $\begin{array}{l}\text { Crenar } \\
\text { chaeot } \\
e\end{array}$ & $\begin{array}{l}\text { ABZ0734 } \\
5.1\end{array}$ \\
\hline
\end{tabular}




\begin{tabular}{|c|c|c|c|c|}
\hline & & $\begin{array}{l}\text { GAACTATTTTGATTTTATATTCATAGATGCCGAT } \\
\text { AAGGAAAGTTACATAGAGTATTTCGATTTGTGC } \\
\text { CTGCCCCTGGTGCGTAAGGGGGGAATAATAG } \\
\text { GCGCCGATAACATACTATTCCCTGAACGTTTTA } \\
\text { ACGAAATGATGGCCGATTACTTATCCCATGTC } \\
\text { CGTAGTAAACCCAATGTCCAGTCAGTCACCAT } \\
\text { CCCGATTGATAACGGGGAAGAAGTAACCATAA } \\
\text { AAATATCAGAGTAA }\end{array}$ & & \\
\hline $\begin{array}{l}\text { PL_02_G } \\
4\end{array}$ & MyOMT & $\begin{array}{l}\text { ATGGGTAAGACGATTCTGGGCCTGGACAGCCA } \\
\text { ATTGTACCAATACCTATTATCAGTGAGCCTAAG } \\
\text { AGAGCCAGAAGTCTTGGTAAAATTGCGTCAAG } \\
\text { AGACGGATAAGTTGCCGATGAACAGAATGCAA } \\
\text { ATCTCACCCGACCAGGGCCAATTCATGAGTTT } \\
\text { GTTAGTTCAGCTAATGGGCGCGAAAAAGACCT } \\
\text { TGGAAATTGGTGTTTTTACTGGATATTCAAGTC } \\
\text { TTGCGGTGGCGCTTGCGCTTCCGCCAGATGG } \\
\text { CAAGATCATAGCGTGTGACGTCGATGAGAAGA } \\
\text { CAACCGCCATTGCAAGGAAATACTGGCAGGAG } \\
\text { GCAGAAGTATCTCACAAAATCGACTTGAGAATA } \\
\text { GCACCCGCACTAGAGACACTTGATAAGTTGAT } \\
\text { TGCGGCAGGAGAAGCGAATTCCTTTGATTTTG } \\
\text { CCTTCATTGACGCTGACAAGAGAAATTATCCG } \\
\text { AACTACTATGAAAGGTGCCTTCAACTGATAAG } \\
\text { GCCGGGTGGCGCTATCGCCATCGACAATGTG } \\
\text { CTGTGGAGCGGGTCAGTGGCCGATAGAAATC } \\
\text { CCACCGATAAAAGGACTATTGCTTTACAGCAAT } \\
\text { TTAATCAGCAATTGCACCAGGACAAAAGGGTG } \\
\text { AAAATCAGTATGTTACCTATAGCGGATGGCCTT } \\
\text { ACATTGGCTATGAAACAAGTAAATTAA }\end{array}$ & $\begin{array}{l}\text { Myxosa } \\
\text { rcina }\end{array}$ & $\begin{array}{l}\text { WP_0364 } \\
84009.1\end{array}$ \\
\hline $\begin{array}{l}\text { PL_02_G } \\
5\end{array}$ & NcOMT & $\begin{array}{l}\text { ATGGAGACCAGTGAACTGGATCGTTCTAAAAT } \\
\text { TTACAAATCCTGGGAAGTAGATGGGAAATTTCA } \\
\text { TAGTGACGGGAGAGAAGTCACCTTGCTAGGGT } \\
\text { ACGTATATAGTCGTCCCGATATCGACCAACTTA } \\
\text { GAGGCTCACCACAAAAAGTACTGGAAGCGATA } \\
\text { GATGACTTTGCGCGTAGCGTGACTGGTCTGAT } \\
\text { CAATATAGGCGAAGCAAAAGGTGCAGTGGTTA } \\
\text { CCAAGCTAATTGCCGATTGCAAGCCAGCAACT } \\
\text { ATTCTGGAACTTGGCGGTTACGTTGGTTACTC } \\
\text { AGCGATTTTGTTCGGCGATGCGTTACGTAAAG } \\
\text { CGGGAGGAAAACAGTACCATAGTGTTGAAAAA } \\
\text { TCCCCCTTATTTGCTGCCGTGGCTGCTTCTCT } \\
\text { GGTCGATCTTGCTGGTCTTAGGGACGTTGTGA } \\
\text { GGATTACAGTAGGGACAGGGGCAGAGGGAAT } \\
\text { CCAGAGATTATACGACTCCGGAGTATTGAAAA }\end{array}$ & $\begin{array}{l}\text { Neuros } \\
\text { pora } \\
\text { crassa }\end{array}$ & $\begin{array}{l}\text { XP_9624 } \\
35.3\end{array}$ \\
\hline
\end{tabular}




\begin{tabular}{|c|c|c|c|c|}
\hline & & $\begin{array}{l}\text { GTCAACTGAGCATGGCCTTTTTCGACCATCATA } \\
\text { AACCTTCTTACACTAGCGATTTGAAGCGTTGC } \\
\text { GAGCGTCTGGGTCTAGTCGGACCAGGTACTGT } \\
\text { GCTTGTGGCTGATAACATGATCCTTCCGGGGA } \\
\text { ATCCCCCCTACGCTGAATGGGTAAGAGCATCC } \\
\text { GTTGCTGAAAAGCAAGAGTTGGACAAAAATGC } \\
\text { GGAAGAAAAGGGATCCCCCAATTTACAATATA } \\
\text { AAAGTAGGGCAATTAAATCATGGGAACCCTCT } \\
\text { GGGCATGAAGACGCCCTAGAGATTACCGAGT } \\
\text { GTGTAGGAATAGAAGCATAA }\end{array}$ & & \\
\hline $\begin{array}{l}\text { PL_02_G } \\
6\end{array}$ & ThOMT & $\begin{array}{l}\text { ATGATCGACGAAAAGATTCAGCACGTGCTGTC } \\
\text { ACGTCTAGAGAAAGACATAGATTACGAAACCG } \\
\text { AACATCTTGGGGAGATTCCTAGTGAGAAACGT } \\
\text { CTGAACTGTATCTCCAAAAACATCGGCATGTTC } \\
\text { TACAACATATTGCTAAAGTCAATTAACGCAAAA } \\
\text { AACATCCTAGAAATAGGGATGTCCGTTGGATA } \\
\text { CTCCGGTCTTTGGTTTGCAGATGCGCTGATGA } \\
\text { GCAATAAACAACTAAACGGCCAGATAATAACAA } \\
\text { TTGACAGAGAGAAATTTAAGATCGACTCTGCAA } \\
\text { GGCAAAATTTTGAAGAAGCAGGGGTGGATTCC } \\
\text { CTAATCAAAATTAGAGAAGGGGAGGCCAAGAA } \\
\text { CATTTTAAGGGAGATCAAGGATGAGTTTAATGA } \\
\text { GAATTACTTTGATTTTATATTTATAGACGCCGAT } \\
\text { AAGGAATCATATATTGAATACTTCGATCTATGC } \\
\text { CTACCACTTGTGAGGAAAGGCGGCATCATTGG } \\
\text { AGCCGATAACATCCTGTTCCCTGAAAGATTTAA } \\
\text { TGAGATGATGTCCGACTACCTGTCACATGTGA } \\
\text { GGAGCAAATCTAATGTCCAATCTGTAACGATAC } \\
\text { CAATTGATAACGGCGAGGAAGTAACCATTAAA } \\
\text { TTGTAA }\end{array}$ & $\begin{array}{l}\text { Thaum } \\
\text { archae } \\
\text { ota }\end{array}$ & $\begin{array}{l}\text { AlF14211 } \\
.1\end{array}$ \\
\hline $\begin{array}{l}\text { PL_02_G } \\
7\end{array}$ & PiOMT & $\begin{array}{l}\text { ATGCCTCCCCGTGTCGCCACAACAAAACCCGG } \\
\text { TCCGCCTCCCTACGATGAGTTAGTTCGTATAG } \\
\text { GCGAGAAGTGTCTTGCATTCGTACACGAAAAC } \\
\text { GCAAAGGAAGGGGATCCCAAGTCTGTGTTAGA } \\
\text { AGCTATTGACACCTTTGGTTATGAACATCATTG } \\
\text { GATGATGAATGTGGGCGACGTCAAGGGGGCC } \\
\text { TTAATAGACGAGGAACTGGAGAAAGCCAAACC } \\
\text { GAAAATTCTTGTAGAACTGGGTGGCCATTTAG } \\
\text { GGTATTCTGCTGTGAGGTTTGCGAGTAAGATG } \\
\text { AGGGAGGTGTCCGGTCCGAGTGCTCATGTTTA } \\
\text { CTCTTTTGAAATTAGTTCAGATTTCGCAGCTAT } \\
\text { AGCAACCAAAATGATAACCTTTGCCGGACTGT } \\
\text { CAGACATGGTAACGATTATTTTGGGAACATAC } \\
\text { GGTAATAATTACAAGAAGCTTAAAGAGAAAGG }\end{array}$ & $\begin{array}{l}\text { Phytop } \\
\text { hthora } \\
\text { infesta } \\
\text { ns }\end{array}$ & $\begin{array}{l}\text { XP_0028 } \\
99214.1\end{array}$ \\
\hline
\end{tabular}




\begin{tabular}{|c|c|c|c|c|}
\hline & & $\begin{array}{l}\text { AGTTGAGCATGTCGATGTGTTTTTTATCGACCA } \\
\text { CGACAAGAAGCTATATAAGTCTGATCTTATCGT } \\
\text { AATCGAACAATCTGGCATGCTTAGGTCCGGGA } \\
\text { GCGTGGTCGTGGCCGACAATGTCGTATATGCA } \\
\text { CACATATCAGATTATGTTGATTACGTTAGAGGA } \\
\text { CATCCCCATTTCAAGTCAGTTCTTAGGGAATCA } \\
\text { CTGCTGGAGTATTCCGACTTGAAAGATGCTAT } \\
\text { CGAAGTGAGCACTTACGTTGAGTAA }\end{array}$ & & \\
\hline $\begin{array}{l}\text { PL_02_G } \\
8\end{array}$ & $\begin{array}{l}\text { Sy6714OM } \\
T\end{array}$ & $\begin{array}{l}\text { ATGGGCAAAGGAATAACCGGCTTCGACCCATC } \\
\text { TTTGTACTCATATCTGCAAAATATTTCAGCGGA } \\
\text { GGAAAGTGTTCACTTAGCGCAACTGAGACGTG } \\
\text { AGACCGCTCACTTACCGGGTGCGTCTATGCAG } \\
\text { ATCACCCCTGAACAGGGGCAATTTCTTGCGCT } \\
\text { GTTGATCGGTCTAACTCGTGCAAAGCGTGTAC } \\
\text { TGGAAATAGGGGTTTTTAGGGGTTATTCTACTC } \\
\text { TTGCTATGGCTCTGCAACTGCCGAGCGACGGA } \\
\text { CAGATCATAGCCTGCGACCAGGATCCCAATGC } \\
\text { TACTGCGATAGCCAAAGAATACTGGCAAAGGG } \\
\text { CGGGCGTCGCCGAGAAAATTTCCCTACGTTTG } \\
\text { GGGCCCGCACTGGCAACCTTAGAGGAACTGG } \\
\text { CCGAGATAAAACCCCTTCCACAATTTGATTTAA } \\
\text { TCTTCATTGATGCTGACAAAAGAAACTACCCAC } \\
\text { ACTATTATGAAGTTAGCCTGAACCTACTTCGTC } \\
\text { GTGGAGGGCTGGTCGTGATCGATAATGTTCTG } \\
\text { TGGCAGGGGAAAGTGGCTGAGGTTGATCCCC } \\
\text { AAGAACCTCAAACGAGAGTATTACAACAGTTTA } \\
\text { ATCGTAATCTGGCTCAAGACGAAAGGGTCAGG } \\
\text { ATTAGTGTTATTCCCCTGGGTGACGGTATGAC } \\
\text { ATTGGCCGTGAAGAGGTAA }\end{array}$ & $\begin{array}{l}\text { Synech } \\
\text { ocystis } \\
\text { sp. } \\
\text { PCC } \\
6714\end{array}$ & $\begin{array}{l}\text { WP_0289 } \\
47825.1\end{array}$ \\
\hline $\begin{array}{l}\mathrm{PL}+02 \_G \\
9\end{array}$ & MyхOMT & $\begin{array}{l}\text { ATGCCGAAATTCACGTTGGTACCCCCTGAGAT } \\
\text { TGAGGCGTATGCTGACGCGCATACAACCGCAC } \\
\text { CCGCACCACTGTTTGACGAATTGAGGGACGTG } \\
\text { ACCTACGCCCGTATGTCTTCTCCAAACATGCA } \\
\text { AGTTGGATCTGTCGAAGGGACCTTCCTGAGAA } \\
\text { TGCTAGTCGCATTAACGGGCGCAAGGAGGGTT } \\
\text { TTGGAAATTGGTACGTTTACAGGCTATTCAGC } \\
\text { GCTGATGATGGCGGAGGCGTTACCAGACGAC } \\
\text { GGCGCGTTGATTACTTGTGATATTGATCCCGA } \\
\text { AGCTACTGATGTGGCGAGAGCCTTCTTTCTGC } \\
\text { GTAGCCCCCACGGGAGAAAAATCGACTTGAG } \\
\text { GATGGGTCCCGCCCTGGATACCTTGAAAACAC } \\
\text { TTCAGGGGCCCTTTGATCTGGCTTTCATCGAT } \\
\text { GCTGACAAGCCGAGTTATGCAGCTTACTGGGA } \\
\text { CGCCGTATTACCGCTATTGCGTCCGGGGGGC }\end{array}$ & $\begin{array}{l}\text { Myxoco } \\
\text { ccus } \\
\text { xanthu } \\
s\end{array}$ & $\begin{array}{l}\text { WP_0115 } \\
55932.1\end{array}$ \\
\hline
\end{tabular}




\begin{tabular}{|l|l|l|l|l|}
\hline & TTAATAATTGCAGACAATGTCTTGTGGAGCGG & & \\
& GAGGGTTCTTCAACCGGAGGGACCCTCCGAC & & \\
& CATGCTATTGTTGATTTTAACGCCAAGGTTGCG & & \\
GCGGACCCAAGGGTCGAGCACGTCCTGTTGA & & \\
CAGTCAGGGATGGAATGATGCTTGCAAGGAAA & & & \\
& AGGTAA & & \\
\hline
\end{tabular}


Figure S1: Growth curves under glucose and lactate conditions for all strains used in this study. For all panels three representative replicates are shown. For all media, both MET and choline were supplemented to a final concentration of $1 \mathrm{mM}$ each. Abbreviations, ETH: ethanol, GAL: galactose, GLC: glucose, GLY: glycerol, LAC: lactate, MET: methionine, THEO: theobromine.

A

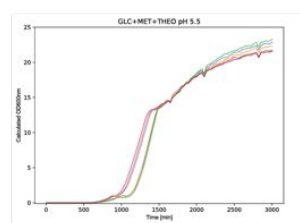

C

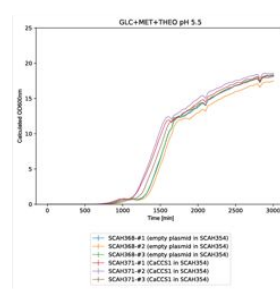

E

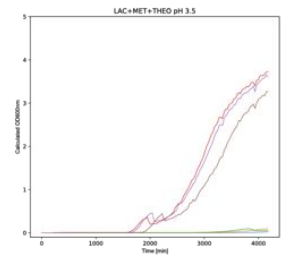

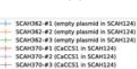

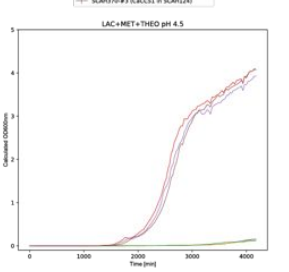

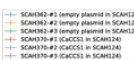

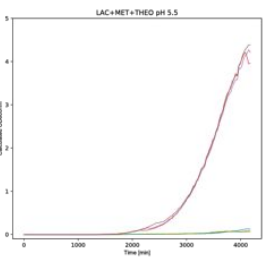

test

G

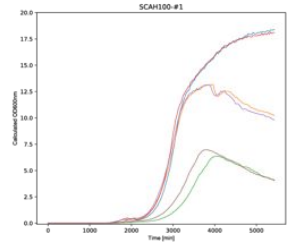

$+2$
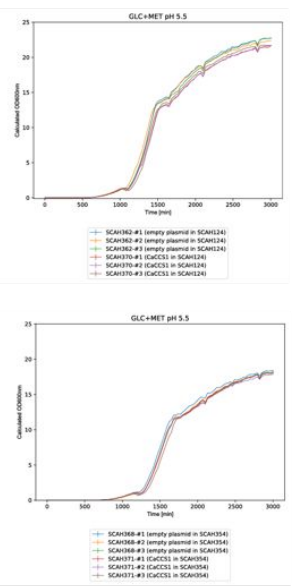

$\mathbf{F}$
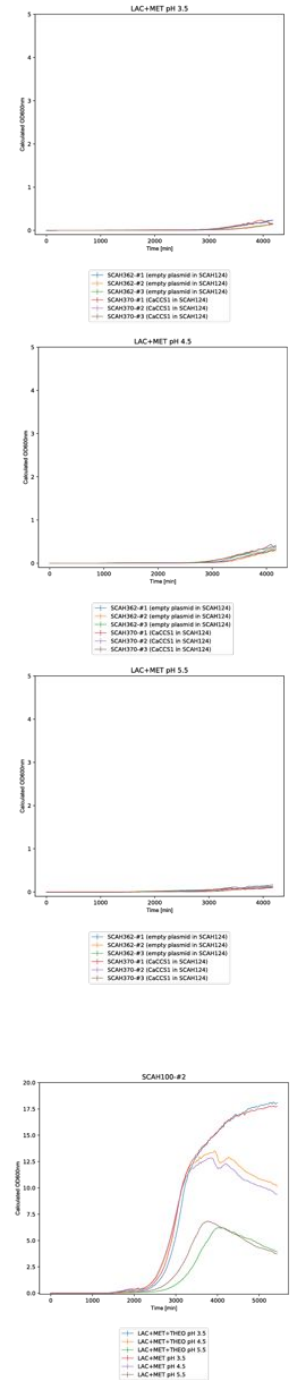
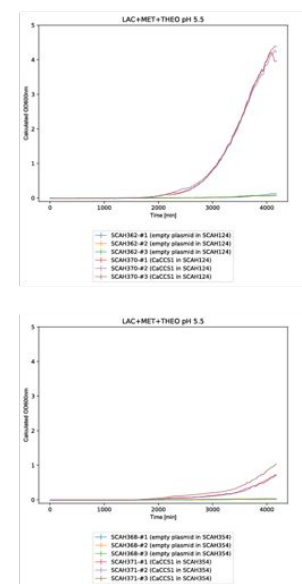

B
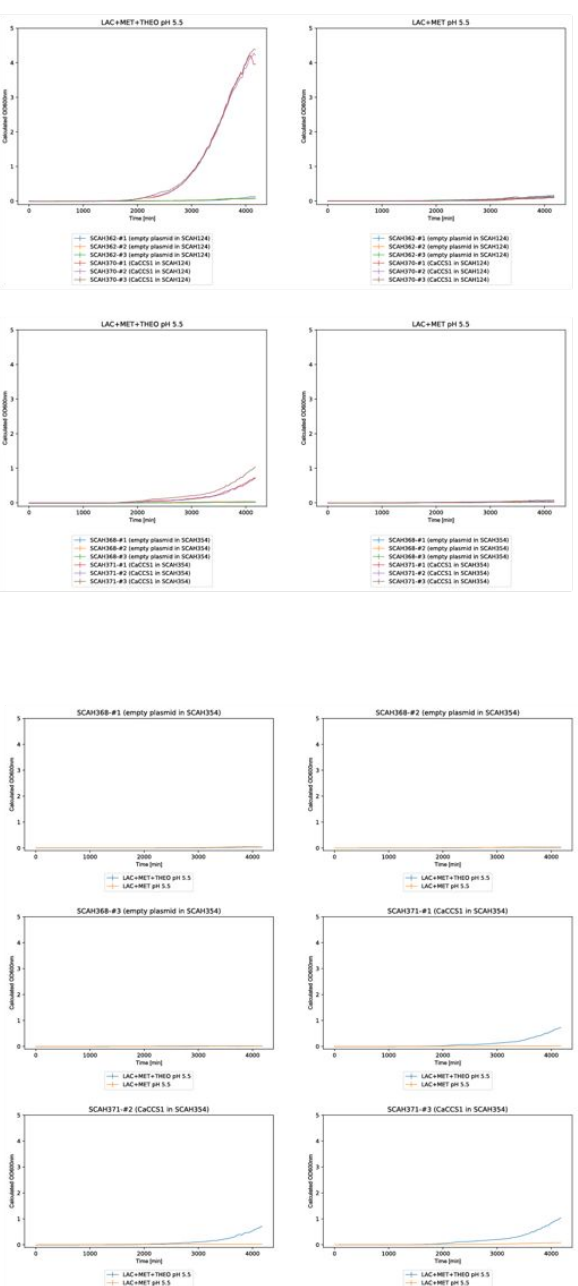
Figure S2: Test of alternative carbon sources. (A) Growth curves of SCAH124 based strains without (SCAH362) and with CaCcs1p expression (SCAH370) cultivated with fermentable carbon sources GAL or GLC. (B) Growth curves of SCAH124 based strains without (SCAH362) and with CaCcs1p expression (SCAH370) cultivated with non-fermentable carbon sources LAC, GLY or ETH in the absence (top) or presence of $0.2 \%$ GLC. For (A) and $(B)$ three representative replicates are shown. For all media, both MET and choline were supplemented to a final concentration of $1 \mathrm{mM}$ each. Abbreviations, ETH: ethanol, GAL: galactose, GLC: glucose, GLY: glycerol, LAC: lactate, MET: methionine, THEO: theobromine.

A
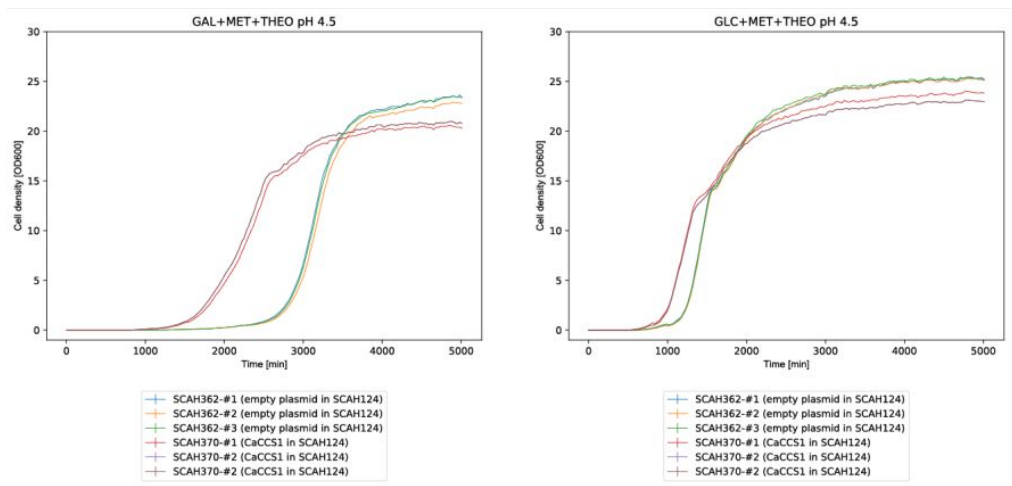

B
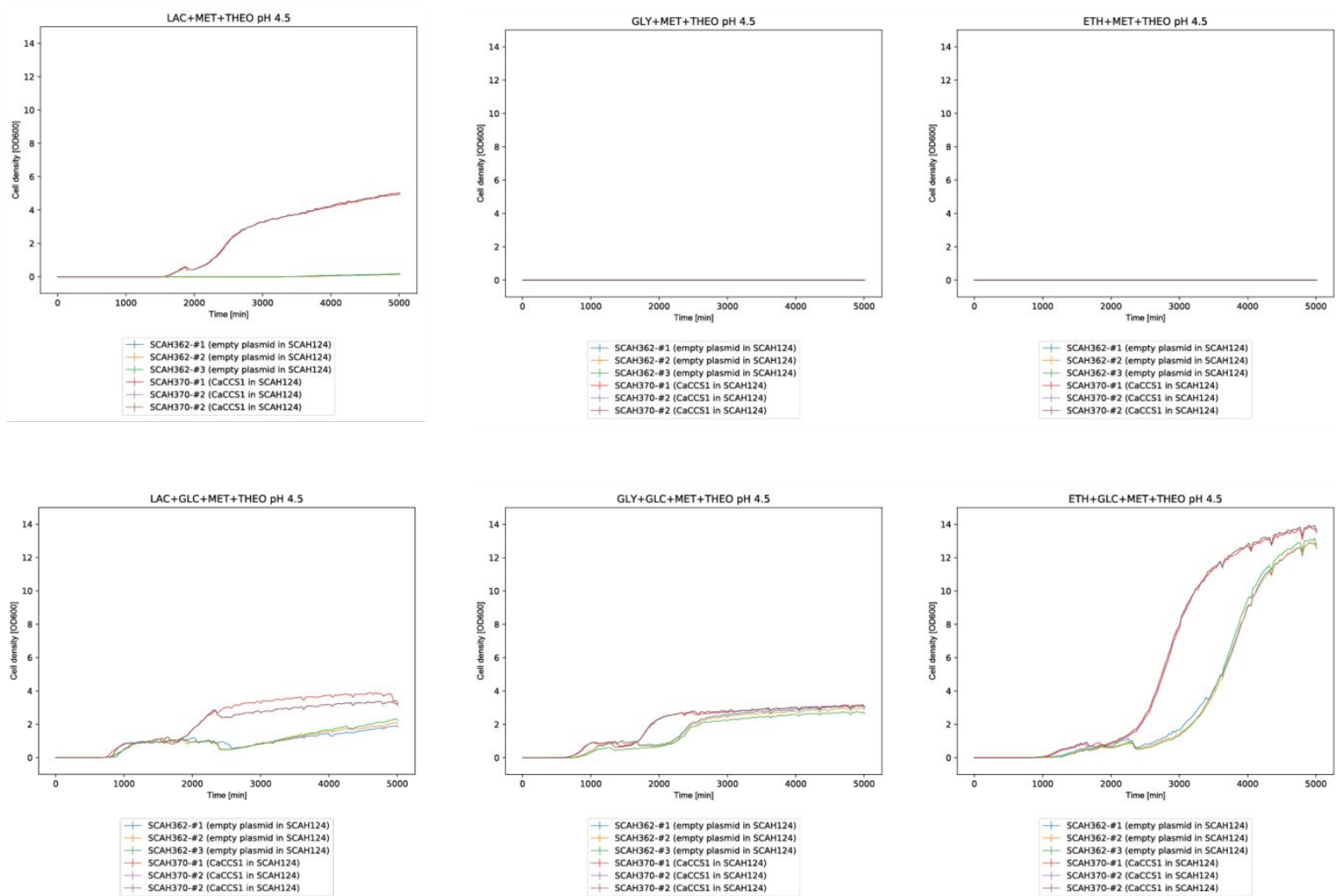